Retraction

\title{
Retraction: Paricio, A.; Lopez-Carmona, M.A. MuTraff: A Smart-City Multi-Map Traffic Routing Framework. Sensors 2019, 19, 5342
}

\section{Sensors Editorial Office}

MDPI, St. Alban-Anlage 66, 4052 Basel, Switzerland; sensors@mdpi.com

It has been brought to our attention that the methods and results presented in [1] are almost identical to those previously published in [2]. The paper [1] will therefore be marked as retracted.

MDPI is a member of the Committee on Publication Ethics and takes seriously its responsibility to publish only high-quality research. We regret that this was not discovered prior to publication and offer our apologies to the readers of Sensors.

\section{References}

1. Paricio, A.; Lopez-Carmona, M.A. MuTraff: A Smart-City Multi-Map Traffic Routing Framework. Sensors 2019, 19, 5342. [CrossRef] [PubMed]

2. Paricio, A.; Lopez-Carmona, M.A. Urban Traffic Routing Using Weighted Multi-Map Strategies. IEEE Access 2019, 7, 153086-153101. [CrossRef] 